\section{(- OPEN ACCESS}

\title{
The reach and adoption of a coach-led exercise training programme in community football
}

\author{
Caroline F Finch, ${ }^{1,2}$ Kathy Diamantopoulou, ${ }^{1}$ Dara M Twomey, ${ }^{3}$ Tim L A Doyle, ${ }^{4}$ \\ David G Lloyd, ${ }^{4,5}$ Warren Young, ${ }^{3}$ Bruce C Elliott ${ }^{4}$
}

\begin{abstract}
${ }^{1}$ Australian Centre for Research into Sports Injury and its Prevention (ACRISP), Monash Injury Research Institute (MIRI), Monash University, Melbourne, Victoria, Australia ${ }^{2}$ Centre for Healthy and Safe Sport, University of Ballarat, Victoria, Australia

${ }^{3}$ School of Health Sciences, University of Ballarat, Ballarat, Victoria, Australia

${ }^{4}$ School of Sports Science, Exercise and Health, University of Western Australia, Perth, Western Australia, Australia ${ }^{5}$ Centre for Musculoskeletal Research, Griffith Health Institute, Griffith University, Gold Coast, Queensland, Australia
\end{abstract}

\section{Correspondence to}

Centre for Healthy and Safe Sport (CHASS), University of Ballarat, SMB Campus, PO Box 668, Ballarat, VIC 3353

Australia;

caroline.finch@monash.edu

Received 15 September 2012 Revised 8 December 2012 Accepted 13 February 2013 Published Online First 12 March 2013

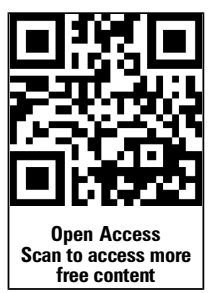

\begin{tabular}{l}
\hline To cite: Finch CF, \\
Diamantopoulou K, \\
Twomey DM, et al. Br J \\
Sports Med 2014;48: \\
718-723. \\
\hline
\end{tabular}

\section{ABSTRACT}

Objective To determine the reach and adoption of a coach-led exercise training programme for lower limb injury prevention.

Design Secondary analysis of data from a groupclustered randomised controlled trial.

Setting A periodised exercise training warm-up programme was delivered to players during training sessions over an 8-week preseason (weeks 1-8) and 18-week playing season.

Participants 1564 community Australian football players.

Main outcome measurements Reach, measured weekly, was the number of players who attended training sessions. Adoption was the number of attending players who completed the programme in full, partially or not at all. Reasons for partial or non-participation were recorded.

Results In week 1, 599 players entered the programme; $55 \%$ attended 1 training session and $45 \%$ attended $>1$ session. By week 12, 1540 players were recruited but training attendance (reach) decreased to $<50 \%$. When players attended training, the majority adopted the full programme-ranging from $96 \%$ (week 1) to above $80 \%$ until week 20. The most common reasons for low adoption were players being injured, too sore, being late for training or choosing their own warm-up.

Conclusions The training programme's reach was highest preseason and halved at the playing season's end. However, when players attended training sessions, their adoption was high and remained close to $70 \%$ by season end. For sports injury prevention programmes to be fully effective across a season, attention also needs to be given to (1) encouraging players to attend formal training sessions and (2) considering the possibility of some form of programme delivery outside of formal training.

\section{INTRODUCTION}

Recently, interest has increased in the delivery of targeted exercise programmes for lower limb injury prevention through coach-led training sessions, particularly in team ball sports. There is strong evidence from randomised controlled trials (RCTs) and/or lab-based biomechanically focused studies that such injuries can be prevented through targeted training incorporating structured warm-up, balance training, side-stepping/cutting skills and jump/landing training. ${ }^{1-15}$

These programmes should be delivered by coaches to teams during a group training session if they are to reduce lower limb injuries in players. Despite the level of scientific evidence, many coaches appear to be unaware of the benefits of these exercise training programmes and do not deliver them to their players. ${ }^{16}$ Recent club/teambased implementation studies, particularly in soccer, demonstrate the limited success of these programmes in actually preventing injuries largely because of 'non-compliance' with the trialled interventions. ${ }^{7} 15$ 17-20 This has been attributed to a range of coach-delivery factors, players' low adherence to the exercises, a perceived lack of relevance of the programmes to community sport, and poor transferability across implementation contexts. ${ }^{19}$ Moreover, players have little motivation to undertake the training programmes if they do not perceive performance benefits. ${ }^{21}$

Commentators have argued that one of the keys to sports injury prevention is compliance with an intervention, ${ }^{22}{ }^{23}$ but only one previous study ${ }^{20}$ has explored intervention compliance in detail. In the context of delivery of the FIFA $11+$ programme, that study reported a team compliance rate of $77 \%$ of sessions and a player compliance of $79 \%$. The study also showed that players with high compliance levels had a significantly reduced risk of injury but compliance was summarised across the season negating the ability to assess weekly variances.

A variety of terms have been used interchangeably to define the success of intervention delivery, including compliance, adherence, and adoption and application of the RE-AIM framework for assessing all aspects of intervention delivery, and success has been advocated. ${ }^{21} 2425$ Two key concepts from the RE-AIM Framework are: (1) intervention reach, or the proportion of the target population that participated in the intervention (similar to Soligard et al's ${ }^{20}$ team compliance), and (2) intervention adoption, or the proportion of people who adopt the intervention (similar to Soligard et al's ${ }^{20}$ player compliance).

We aimed to report how the RE-AIM Framework components of reach and adoption of an exercise programme change across preseason to the playing season in community Australian football. Reach was assessed in terms of the overall numbers of players recruited to an RCT, and in relation to the varying recruitment level, given that recruitment did not occur at a single time point.

\section{METHODS}

This study is a secondary analysis of data from a group-clustered RCT of an exercise programme in community Australian football: The Preventing Australian Football Injuries through eXercise (PAFIX) project. Full details of the study design, data collection protocol and accuracy of the data 
collection methods are published elsewhere. ${ }^{26} 27$ Briefly, PAFIX was a two arm group-clustered RCT conducted in community Australian football during the 2007 and 2008 playing seasons. Eighteen clubs from two Australian states nominated 40 teams and these groups of players were randomised to one of two intervention arms. The study was approved by the University of Ballarat and University of Western Australian Ethics Committees.

Overall, 1564 players were progressively recruited into the trial from 8 -weeks preseason until week 5 of the playing season. This phased recruitment strategy was necessary to include players with reasons for unavailability during the preseason and the intent was to expose as many players as possible to the intervention. Recruitment concluded at week 5 of the season to coincide with the end of the first in-season section of the periodised programme, as it was considered unlikely that there would be any training benefits for players not involved with the training up to then. The delivered intervention was 26 weeks long (8-week preseason and 18 in-season weeks). The programme was designed to take place for approximately 10-20 min during the warm-up time within the training session.

To be included in this secondary data analysis, players were required to be aged at least 18 years by the start of the 8 -week preseason period and to have attended $>1$ training session by week 5 of the in-season period. As per usual football practices, players were expected to attend two training sessions a week, but not all did so.

Player training attendance was recorded at each training session on a standardised form (figure 1). Reasons why players did not fully participate or only partially participated in the training programme for each training session they attended were noted when known.

\section{Reach}

For each of the 26 weeks, the number of training sessions attended by each player was categorised as either: one training session attended; $>1$ training session attended or no (0) training sessions attended. Reach was defined as the number of players who attended training in a given week and was computed as the sum of players in the first two categories. Reach was calculated weekly and presented in one of two ways: (1) as a percentage of the total cohort of registered players and (2) as a percentage of the number of players registered by that week (eg, players who did not start in the study until week 5 were not included in the denominator for the week 3 reach).

To test whether there were significant changes in reach across the preseason to end of season, a Generalised Estimating Equation (GEE) model, based on a logistic regression model with repeated measures adjustment and cluster adjustment, was fitted to the weekly training attendance data. The dependent variable was the training attendance for each player during each week. The week of training attendance was included as a factor in the model, while a nested term of a football club effect nested within the state (ie, state (club)) was included to account for design/sampling clustering effects. For the trend analysis taking into account the number of players recruited at any given week, the model was fitted to the weekly training attendance with the number of players entering the programme each week included as a separate 'number of players' variable.

\section{Adoption}

The level of player participation in the training programme for those who attended training each week, or adoption, was recorded as participated fully in the programme, only partially participated or did not participate.

A GEE model, based on a logistic regression model with repeated measures and cluster adjustment, was fitted to the weekly adoption participation. The dependent variable was the programme participation status (any or none) for each player during each week. The week of programme participation was included as a factor as was a nested term state (club) to account for design clustering.

\section{RESULTS}

Overall, 1564 football players met the inclusion criteria and their weekly training attendance (programme reach) varied
Figure 1 Data recording sheet for player training attendance and level of programme participation.

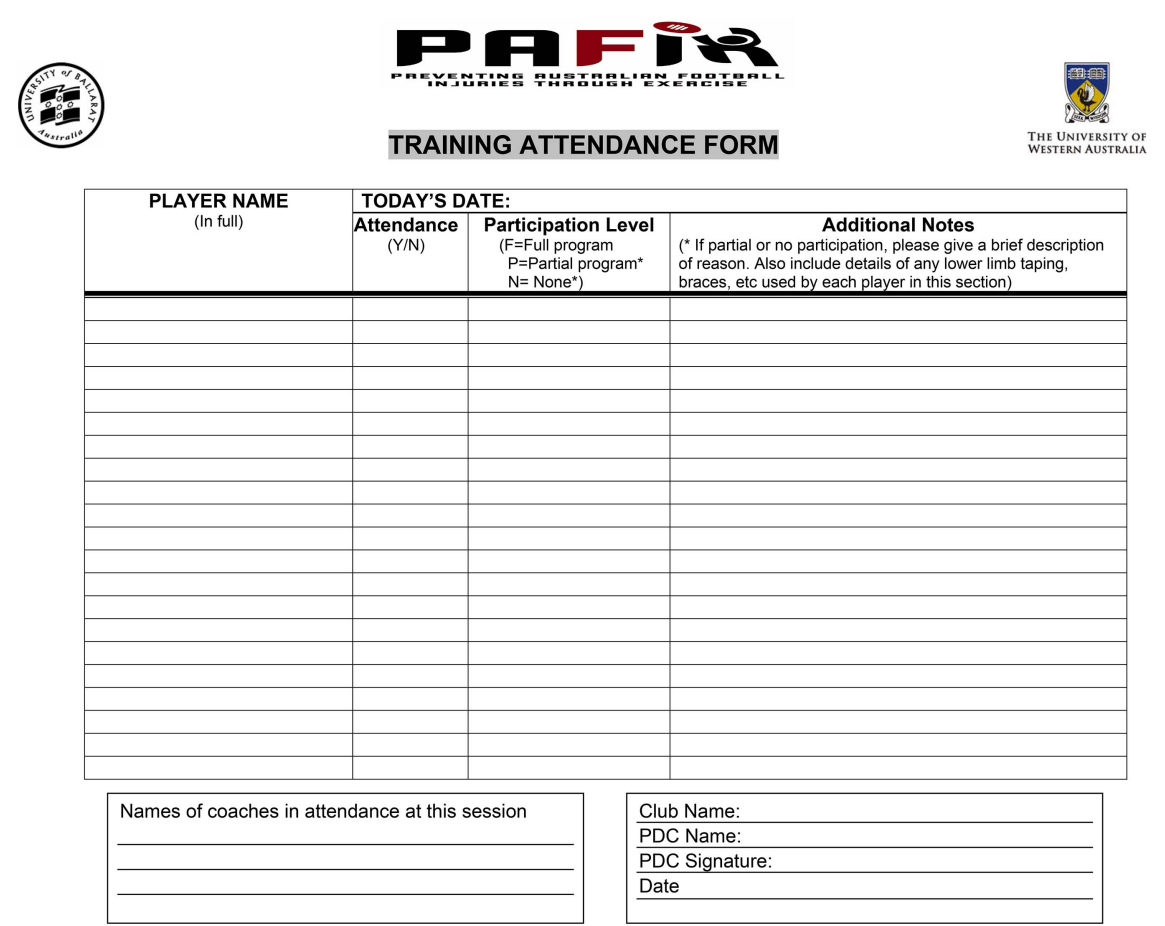


Figure 2 Training attendance per week by football players - as a proportion of the entire cohort of 1564 players.

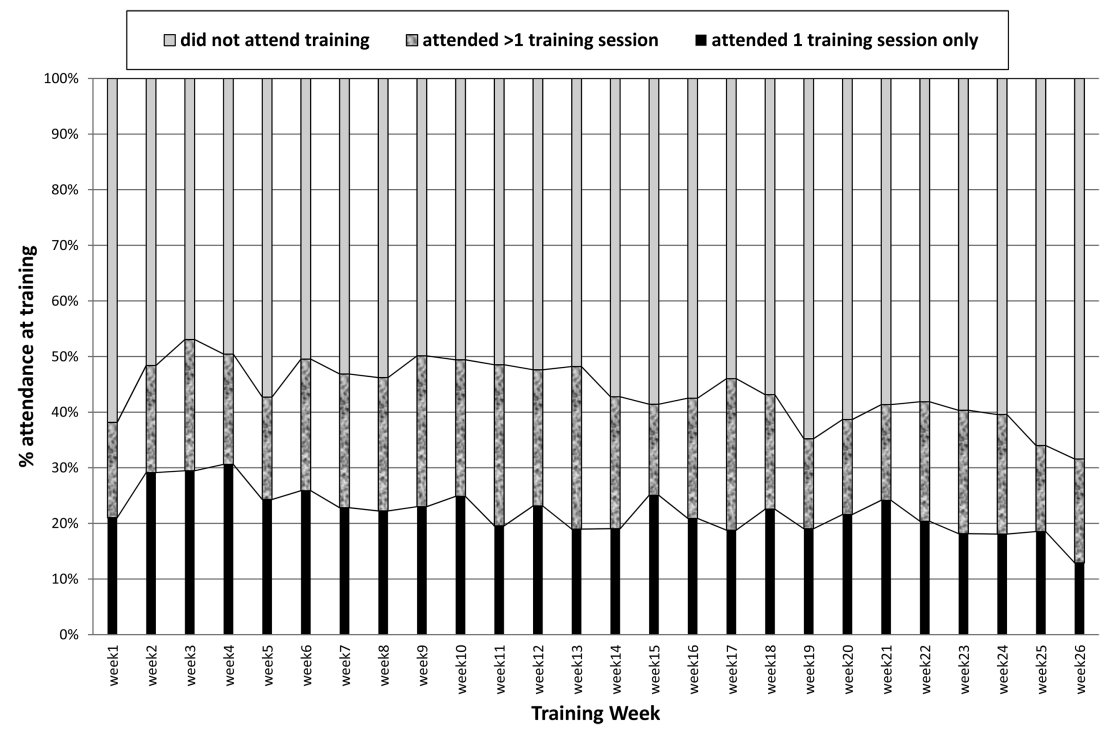

The majority of players participated in the full programme each week (figure 4). Full programme participation ranged from $96 \%$ in week 1 to $64 \%$ in week 24 . Full or partial programme participation was almost $98 \%$ in week 1 and remained above $80 \%$ until week 20 when programme participation dropped to $72 \%$. This corresponded to a significant reduction of $27 \%$ $(\mathrm{p}<0.001)$.

Reasons for partial participation or non-participation in the programme were recorded for 849 players over 4638 player training sessions (table 1). The top four reasons for partial or nonparticipation collectively accounted for $93.3 \%$ of all reasons.

\section{DISCUSSION}

The success of every injury prevention measure requiring behaviour change depends upon the target audience being aware of it and then deciding to adopt it. In the RE-AIM Framework, ${ }^{28} 29$ this corresponds to ensuring high reach and adoption. Maintained behavioural change over time is required to ensure that the injury prevention benefits are sustained and realised in reduced injury rates.
Figure 3 Training attendance (reach) per week by football players-as a proportion of the players who had entered the training programme each week.

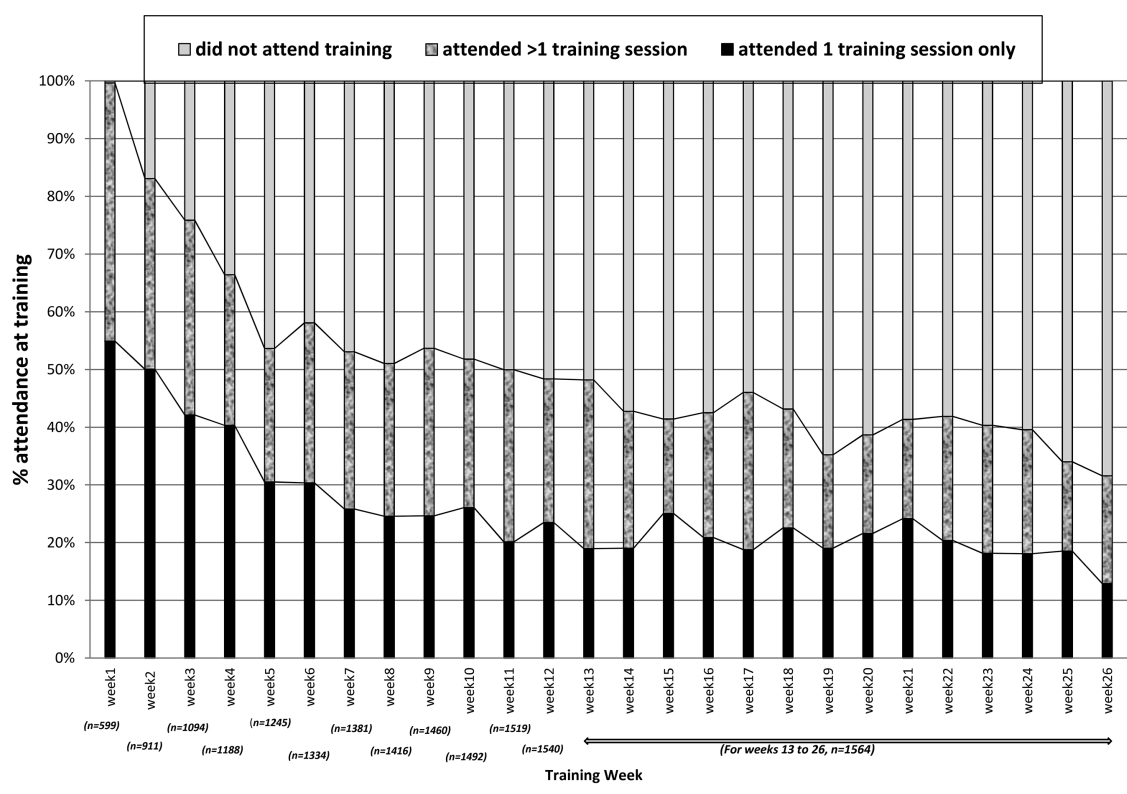


Figure 4 Adoption or programme participation for players attending at least one training session per weekas a proportion of players who attended training in a given week.

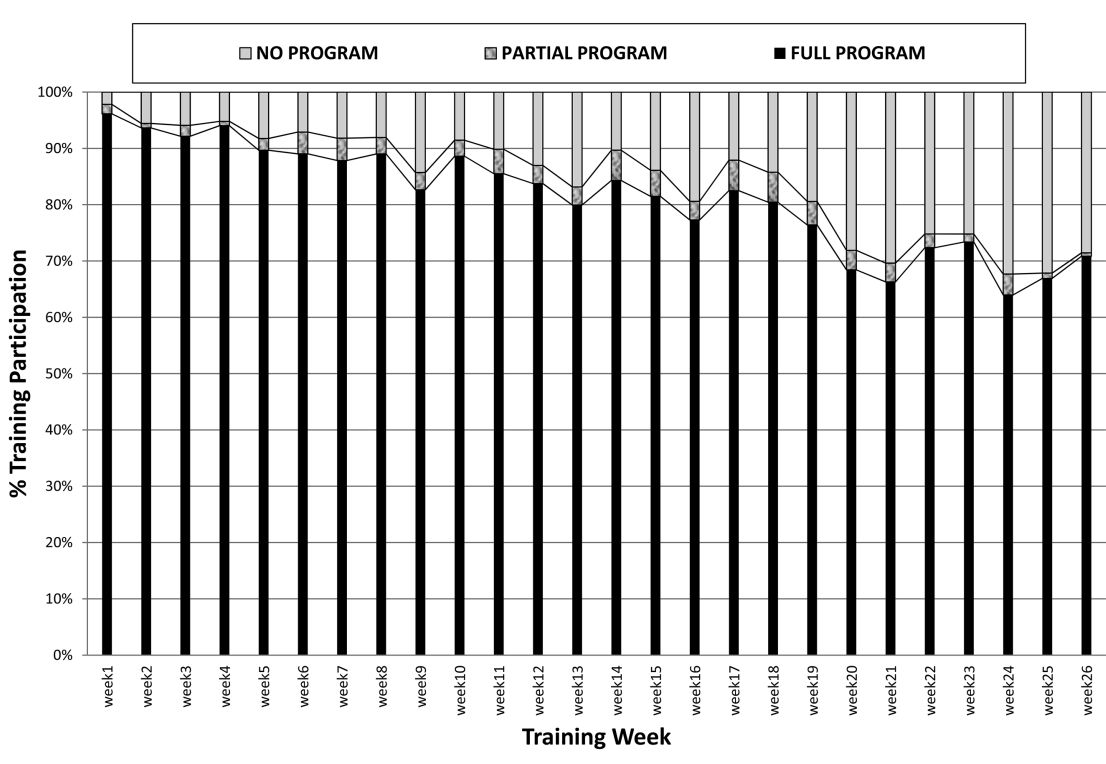

Only very recently have studies considered whether or not players actually adopt training interventions when delivered in team sport settings by coaches. ${ }^{15}$ 17-20 This suggests a major gap in the literature, and without this information, clear explanations for why training programmes do/do not prevent injuries will continue to be limited. ${ }^{21} 25$ Furthermore, a lack of efficacy/ effectiveness evidence for exercise programmes prior to their being delivered in team sport means that if the training fails to prevent injuries, it will be unclear if this outcome is due to the type of exercises or low reach/adoption.

This paper presents a secondary analysis of data from an effectiveness trial for the delivery of a coach-led exercise training intervention in community Australian Football. Specifically, it describes the reach and adoption of exercise programmes delivered at community football training sessions over a preseason period and subsequent playing season. These two intervention components provide important information about the challenges that need to be addressed when delivering exercise programmes through training sessions only.

Reach was defined as the number of players who attended each training session because they were the only people that the intervention was delivered to, and hence who it could potentially reach. If players did not attend training, they would not be 'exposed' to the intervention in any other manner, as there was no other way for the players to receive the training guidance from their coach. At most, 70\% of players attended training sessions in any given week and more generally, it was between $50 \%$ and $70 \%$. Most players who attended training did so only for one session per week. This attendance pattern reflects the sporting culture and local influences in each region. Community football usually has two training sessions a week, with the coach deciding upon the final team composition at the Thursday training session. It is possible that some players only attended training on the Thursday to maximise their chances of being selected to play on the weekend. In the Victorian sample, the league was based in a large regional centre and a proportion of the players worked in a metropolitan city about $100 \mathrm{~km}$ away, but returned to the region to play football. It is possible that up to one-third of the contracted players from each club would only attend local training once a week, and train in the metropolitan city for the other session, irrespective of whether or not they were selected to play. Others may have stopped training altogether never going to be selected to play. In the Western Australian sample, some players worked in remote locations and therefore spent some time away from the metropolitan centre during the season due to work commitments.

Training attendance tended to decline towards the end of the playing season, presumably reflecting the fact that not all teams made the finals series. There was no evidence of a significant increase in the rate of injuries towards the end of the season that could explain this result (unpublished data). Initial increases in the numbers of recruited players, which lead to increased reach, also reflect a common situation in community football where many players are still playing in community cricket competitions (the predominant summer sport) and are not able to take up football training until their cricket commitments have been completed. In the Victorian sample, the regional league was in a 'university town' and some of the observed increase in numbers of players in the preseason could have been influenced by the return of university students. Similarly, the observed dip in numbers at week 19 may have been due to students tending around the start of the season when they realised that they were

Table 1 Top four reasons for partial or no participation in the exercise training programme during weekly training sessions

Reason for partial or no participation

Per cent

(n)

Programme not run (most commonly because the programme was in maintenance or a scheduled break phase) 
to return home during the university break. Unfortunately, we were not able to record why players did not attend specific training sessions and so these suggestions are based on anecdotal observations only.

The varied availability of players across a football season, particularly during the pre-season, has an impact on measures of intervention reach. In the community football context, by definition, reach will be low at the start of the pre-season when there are fewer players available. This indicates that computing preseason reach as a function of the final cohort size will give a biased picture, as shown by a comparison of figures 1 and 2 .

Adoption was defined as the number of players who attended training who also participated in the exercise programme. Irrespective of reach, adoption was generally high among players who attended training sessions. During the pre-season (weeks 1-8) and into the first half of the season, adoption was particularly high, with the vast majority of players who attended training undertaking the full programme. However, there was a significant decline in the adoption over the season, with low levels in the last 7-9 weeks, which may have been related to the team's finals series hopes. It is possible that adoption decline during the last few weeks could have been due to the maintenance phase of the programme being unable to sustain player interest. While reasons for partial or non-participation were recorded, this was inconsistent across the season and incomplete for many players. Nonetheless, it is apparent that the most common reasons for non-adoption were the programme not being delivered, such as could occur during a maintenance or RCT testing session, players either arriving late, needing to leave early or a player experiencing injury or soreness.

A major strength of this study was that training attendance and programme participation was reported prospectively on a standardised data collection form by trained primary data collectors (PDCs) who were paid to attend each training session. These PDCs were observed during each session, but unless they spoke to specific players, the recorded reasons for non-participation were not confirmed. The PDC observations were maintained over 26 weeks, leading to the longest series of both session-specific weekly attendance and participation data yet reported in community sport.

This paper has shown that monitoring the reach and adoption of injury prevention interventions on a weekly basis throughout a season is more informative than just reporting a single figure over an entire season. There is now a need for future studies to also examine the reasons for different levels of reach and adoption to better identify opportunities for removing barriers to intervention uptake.

\section{Implications for prevention}

Based on this detailed recording and analysis of player attendance at training sessions, it can be concluded that interventions that are solely delivered to players through their attendance at training sessions will only reach $50-60 \%$ of all targeted players. It is not surprising, therefore, that the level of benefit observed from coach-led exercise training programmes has been reportedly low, when only half of the targeted players are exposed to the intervention.

Future implementation delivery plans designed to involve coach-led activities during coaching sessions will need to include strategies to maximise training attendance, as well as strategies to get players to do exercises once they arrive. This will require a major change in culture at many local football clubs and research is needed to describe current culture and to identify drivers of actions, or barriers towards addressing them, for both coaches and players. As players think that training should focus more on performance than on injury prevention, ${ }^{21}$ this view would need to be considered. Coaches would also be more motivated to deliver these warm-up programmes during training sessions if they led to better team performance. ${ }^{16}$

Given the low attendance at training, perhaps there is a need to consider supplemental strategies to reach the $40-50 \%$ of players who do not attend training. Future exercise programmes could consider including different intervention dissemination strategies for players unable to attend training sessions. Of course, some of those will not want to do training of any form, but there could be scope to reach more players through the development of guidance programmes (eg, Smartphone apps, websites, social networks, etc) to support those players to undertake exercises to reduce their football injury risks. There is still a need for research into the value of unsupervised delivery of sports injury prevention programmes (eg, home programmes with and without additional guidance) to identify the most promising alternatives for players who are not reached through regular training attendance.

A positive aspect is that if players attend training, they will adopt programmes delivered by their coaches. However, there is still a challenge in programme delivery in that different players will commence their season/preseason at different time points and programmes may need to be individually targeted for these players.

Notwithstanding the best efforts to increase both programme reach and adoption from the start of a season, this study has shown that both significantly decline over the season. This has important implications for the long-term maintenance or sustainability of sports injury prevention programmes, an area that has only had scant attention paid to it in the literature to date. $^{24} 25$ There is an obvious need for well-designed implementation studies to answer questions such as: how can programmes be designed and delivered to maintain player interest over a season? Is having just one programme for all parts of the season the best way to deliver injury prevention programmes in community football? Is there a best way to manage progressions in an exercise programme over its delivery across a playing season? Given the decline in both reach and adoption over a season, would it be more effective to concentrate all preventive efforts in only the preseason period? Given that many players are unable to attend two training sessions a week, should programmes be designed to be effective when delivered at only at one training session per week?

Finally, to ensure maximal injury prevention benefit from participation in exercise training programmes, it is essential that the quality (or fidelity) of exercise is appropriate. This would be expected to require proper coaching supervision during programme delivery to ensure that the players are performing the exercises with correct technique and to the required level of intensity and repetition.

What this study adds

- This study provides a means of presenting data on the reach and adoption of sports injury prevention programmes that is consistent with the RE-AIM Framework.

- The study shows that interventions with a sole delivery mode through organised training sessions will only reach $50 \%$ of target players.

- However, if players attend training, they tend to adopt the injury prevention programme delivered by their coach. 
Contributors CFF, DGL and BCE contributed to the conception and design of the study that the data came from. CFF conceived the secondary analysis in this paper study, contributed to the analysis plan and had major responsibility for the writing of the paper. KD undertook data coding and analysis, developed the analysis plan and contributed to the writing of the paper. DMT and TLAD coordinated the data collection phase, developed the data collection tools, designed the training programme components and contributed to the writing of the paper. DGL and BCE both provided editorial input into the paper writing. WY contributed to the interpretation of the data and provided editorial input into the writing of the paper.

Funding The PAFIX study was funded by a nationally competitive research grant from the (Australian) National Health and Medical Research Council (NHMRC)— Project ID 400937. The Australian Centre for Research into Injury in Sport and its Prevention (ACRISP) is one of the International Research Centres for Prevention of Injury and Protection of Athlete Health supported by the International Olympic Committee (IOC). CFF was supported by an NHMRC Principal Research Q10 Fellowship (ID: 565900).

\section{Competing interests None.}

Ethics approval University of Ballarat and University of Western Australia ethics committees.

Provenance and peer review Not commissioned; externally peer reviewed.

Open Access This is an Open Access article distributed in accordance with the Creative Commons Attribution Non Commercial (CC BY-NC 3.0) license, which permits others to distribute, remix, adapt, build upon this work non-commercially, and license their derivative works on different terms, provided the original work is properly cited and the use is non-commercial. See: http://creativecommons.org/ licenses/by-nc/3.0/

\section{REFERENCES}

1 Hewett T, Lindenfeld T, Roiccobene J, et al. The effect of neuromuscular training on the incidence of knee injury in female athletes: a prospective study. Am J Sports Med 1999;27:699-706.

2 Lloyd D. Rationale for training programs to reduce anterior cruciate ligament injuries in Australian football. JOSPT 2001;31:645-54.

3 Verhagen E, Van Der Beek A, Twisk J, et al. The effect of a proprioceptive balance board training program for the prevention of ankle sprains: a prospective controlled trial. Am J Sports Med 2004;32:1385-93.

4 Mandelbaum B, Silvers $H$, Watanabe $D$, et al. Effectiveness of a neuromuscular and proprioceptive training program in preventing anterior cruciate ligament injuries in female athletes: 2-year follow-up. Am J Sports Med 2005;33:1003-10.

5 Dempsey A, Lloyd D, Elliott B, et al. The effect of technique change on knee loads during sidestep cutting. Med Sci Sports Exerc 2007:39:1765-73.

6 Emery CA, Rose MS, McAllister JR, et al. A prevention strategy to reduce the incidence of injury in high school basketball: a cluster randomized controlled trial. Clin J Sports Med 2007;17:17-24.

7 Croisier JL, Ganteaume S, Binet J, et al. Strength imbalances and prevention of hamstring injury in professional soccer players: a prospective study. Am J Sports Med 2008;36:1469-75

8 Pasanen K, Parkkari J, Pasanen M, et al. Neuromuscular training and the risk of leg injuries in female football players: cluster randomised controlled study. $\mathrm{Br}$ J Sports Med 2008:42:502-5.
9 Soligard T, Myklebust G, Steffen K, et al. Comprehensive warm-up programme to prevent injuries in young female footballers: cluster randomised controlled trial. BMJ 2008;337:a2469.

10 Dempsey $A$, Lloyd D, Elliott B, et al. Changing sidestep cutting technique reduces knee valgus loading. Am J Sports Med 2009:37:2194-200.

11 Cochrane J, Lloyd D, Besier T, et al. Training affects knee kinematics and kinetics in cutting maneuvers in sport. Med Sci Sports Exerc 2010;42:1535-44.

12 Emery $C$, Meeuwisee W. The effectiveness of a neuromuscular prevention strategy to reduce injuries in youth soccer: a cluster-randomised controlled trial. $\mathrm{Br}$ J Sports Med 2010:44:555-62.

13 LaBella C, Huxford M, Grissom J, et al. Effect of neuromuscular warm-up on injuries in female soccer and basketball athletes in urban public high schools: cluster randomized controlled trial. Arch Ped Adolesc Med 2011;165:1033-40.

14 Waldén $\mathrm{M}$, Atroshi I, Magnusson $\mathrm{H}$, et al. Prevention of acute knee injuries in adolescent female football players: cluster randomised controlled trial. BMJ 2012;344:e3042.

15 Donnelly C, Elliott B, Ackland T, et al. Translating ACL injury prevention research to injury prevention practice: Incorporating the recent evidence. Res Sports Med 2012:20:239-62.

16 Twomey D, Finch C, Roediger $\mathrm{E}$, et al. Preventing lower limb injuries: is the latest evidence being translated into the football field? J Sci Med Sport 2009;12:452-6.

17 Engebretsen $\mathrm{AH}$, Myklebust G, Holme I, et al. Prevention of injuries among male soccer players: a prospective, randomized intervention study targeting players with previous injuries or reduced function. Am J Sports Med 2008;36:1052-60.

18 Steffen K, Myklebust G, Olsen 0, et al. Preventing injuries in female youth football-a cluster-randomized controlled trial. Scand J Med Sci Sports 2008;18:605-14.

19 Kilding AE, Tunstall H, Kuzmic D. Suitability of FIFA's "The 11" training programme for young football players-impact on physical performance. J Sports Sci Med 2008;7:320-6.

20 Soligard T, Nilstad A, Steffen K, et al. Compliance with a comprehensive warm-up programme to prevent injuries in youth football. Br J Sport Med 2011;44:787-93.

21 Finch CF, White $\mathrm{P}$, Twomey $\mathrm{D}$, et al. Implementing an exercise-training programme to prevent lower-limb injuries: considerations for the development of a randomised controlled trial intervention delivery plan. Br J Sports Med 2011:45:791-6.

22 Finch $C$. A new framework for research leading to sports injury prevention. J Sci Med Sport 2006:9:3-9.

23 Van Tiggelen D, Wickes $S$, Stevens $V$, et al. Effective prevention of sports injuries: a model integrating efficacy, efficiency, compliance and risk taking behaviour. Br J Sports Med 2008;42:648-52.

24 Finch $C F$, Donaldson A. A sports setting matrix for understanding the implementation context for community sport. Br J Sports Med 2010;44:973-8.

25 Finch $C$. No longer lost in translation-the art and science of sports injury prevention implementation research. Br J Sports Med 2011;45:1253-7.

26 Finch C, Lloyd D, Elliott B. The Preventing Australian Football Injuries with eXercise (PAFIX) study — a group randomised controlled trial. Inj Prev 2009:15:e1.

27 Twomey D, Finch C, Doyle T, et al. Level of agreement between field-based data collectors in a large scale injury prevention randomised controlled trial. J Sci Med Sport 2011;14:121-5

28 Glasgow R, Vogt T, Boles S. Evaluating the public health impact of health promotion interventions: the RE-AIM framework. Am J Public Health 2001;89:1322-7.

29 Glasgow R, Klesges L, Dzewaltowski D, et al. Evaluating the impact of health promotion programs: using the RE-AIM framework to form summary measures for decision making involving complex issues. Health Educ Res 2006;21:688-94. 